\title{
Efficient All-Optical Wavelength Conversion scheme based on a Saw-Tooth Pulse Shaper
}

\author{
F. Parmigiani, M. Ibsen, P. Petropoulos, D. J. Richardson
}

\begin{abstract}
We experimentally demonstrate the use of sawtooth optical pulses, generated using a superstructured fiber Bragg grating (SSFBG), to achieve high performance and efficient all-optical wavelength conversion in a scheme based on cross-phase modulation (XPM) in a highly nonlinear fiber (HNLF), with subsequent offset filtering. Compared to the use of more conventional waveforms, such as Gaussian pulses, the purpose-shaped saw-tooth pulses allow an improvement in the optical signal to noise ratio (OSNR) of the wavelength converted signal of around $15 \mathrm{~dB}$ as well as a more than $6 \mathrm{~dB}$ improvement in receiver sensitivity.
\end{abstract}

Index Terms - Optical pulse shaping, fiber Bragg grating, highly nonlinear fiber, cross-phase modulation, triangular shape.

\section{INTRODUCTION}

A ll-optical wavelength conversion is likely to represent a key function within future ultra-high speed communication systems since it can be used to avoid wavelength blocking and give flexibility in the network design. The various schemes applicable to return-to-zero (RZ) wavelength conversion so far reported have primarily exploited nonlinear effects in semiconductor optical amplifiers [1] or in fibers. For the latter case, the nonlinear process can be either four wave mixing (FWM) [2], XPM in an interferometric [3] or non interferometric [4] configuration, or self-phase modulation (SPM) if the required wavelength shift from the original signal is modest [5]. Among these possible alternatives, the technique based on XPM-induced sidebands onto a continuous wave (CW) beam (at the desired wavelength), which can then be filtered to provide a wavelength converted representation of the original signal, represents a particularly robust and simple solution. The filtering is usually performed using a bandpass filter to reject the pump signal in conjunction with a narrowband notch filter to reject light at the original carrier frequency. This notch filter gives extra complexity to the system, since it has to be precisely tuned to the $\mathrm{CW}$ wavelength and to be extremely narrowband (of the order of

This work was supported in part by the STREP PHASORS (FP7-ICT2007-2) and FP7 Network of Excellence BONE (FP7-ICT-2007-1 216863). Dr. M. Ibsen acknowledges the support from a Royal Society University Research Fellowship.

The authors are with the Optoelectronics Research Centre, University of Southampton, SO17 1BJ, United Kingdom (e-mail: frp@orc.soton.ac.uk). a few hundreds of pm) in order to avoid any distortion to the spectral envelope of the converted signal. Usually, the tight notch filtering required is implemented using high performance filters, such as a custom-made inline fiber Bragg grating [6], or a loop mirror filter, comprising a short piece of birefringent fiber in a Sagnac interferometer [3]. Both of these forms of filter are subject to drift due to their acute environmental sensitivity. Furthermore, since most of the wavelength conversion schemes to date use either Gaussian or soliton control pulses, which exhibit significant variation in intensity gradient across their profile, they usually produce chirped pulses at the new frequency. The excess bandwidth associated with this wavelength chirp further exacerbates the demands on the filter. Techniques that can reduce the filter tolerances and allow the use of conventional and more robust band-pass filter technologies are thus of significant practical interest.

In [7] we previously demonstrated the advantages of shaping the signal to be wavelength converted into a sawtooth waveform in an SPM-based wavelength conversion scheme. The linear gradient of the saw-tooth pulses ensures that the instantaneous optical frequency shift, which is directly proportional to the intensity gradient of the pump waveform, is constant, thus introducing minimum additional spectral broadening. Furthermore, because of the asymmetry of the shape, the spectral intensity evolution is also asymmetric, with the smoother edge (which has much more power associated to it) experiencing less frequency shift, but much higher and more confined spectral energy peaks than the sharper trailing edge (see [7]). This is in contrast to more conventional (e.g. Gaussian) pulse shapes, which are characterized by a constantly varying intensity gradient and give rise to the typical, symmetrically broadened SPM spectrum. The attraction in shaping the pulses in this manner is that a converted signal of high spectral density and minimal distortion can be obtained. However, when based on SPM, this scheme is restrictive in terms of the wavelength assignment of the converted signal, since the wavelength shifts that can be achieved are only modest. This limitation can be alleviated by using a sawtooth pulse shape for the pump signal in a wavelength conversion scheme based on generating XPM-induced sidebands on a CW carrier. The wavelength shifted spectrum is also far more confined and can readily be filtered from the $\mathrm{CW}$ wave using conventional band-pass 
filters. This gives more flexibility in the choice of the pump/signal wavelength allocations but also allows most of the wavelength converted signal energy to pass through the subsequent offset filter, improving the OSNR and subsequently the overall system performance. Following the preliminary results reported in [8], in this Letter, we experimentally demonstrate, in a proof of principle experiment, a simple and cost effective all-optical wavelength conversion scheme based on XPM spectral broadening induced by saw-tooth pulses and filtering using conventional bandpass filters and show the improved performance achieved as compared to more conventional pulse shapes.

We note that this saw-tooth shape has also been demonstrated to be very interesting for various other applications - amongst these, optical time division add-drop multiplexing is a clear and important example [9-10].

\section{EXPERIMENTAL SET-UP AND RESULTS}

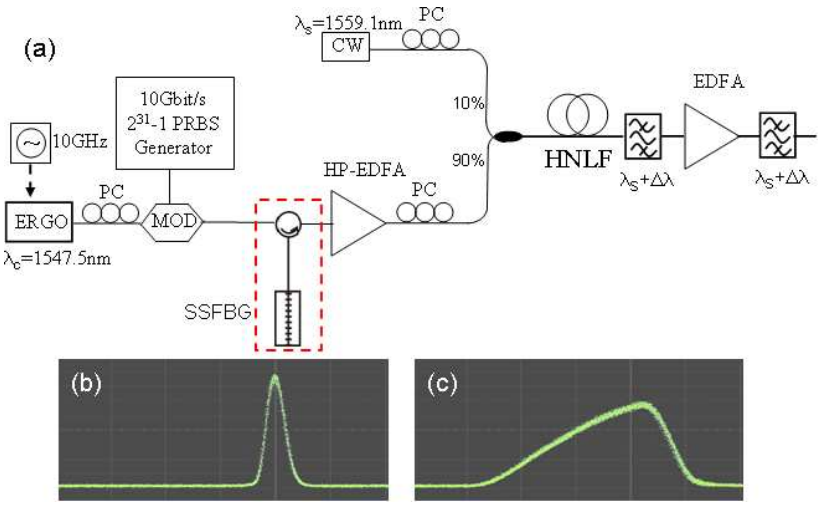

Figure 1. a) Experimental set-up of the wavelength conversion system. EDFA: erbium doped fiber amplifier. b-c) Measured Gaussian and sawtooth intensity profiles using an OSO, time scale: $5 \mathrm{ps} / \mathrm{div}$.

The experimental set-up is shown in Fig.1(a). An Erbium Glass Oscillator (ERGO) operating at $1547.5 \mathrm{~nm}$, which generates $\sim 1.3 \mathrm{ps}$ Gaussian pulses at $10 \mathrm{GHz}$, is used to generate the control signal. The control pulses are amplitude modulated by a $2^{31}-1$ pseudorandom bit sequence (PRBS) using a lithium-niobate Mach-Zehnder modulator (MOD), reflected off the spectrally designed SSFBG to be shaped into 10ps full width at half maximum (FWHM) saw tooth pulses (the full characterization of the grating is reported in [7]) and amplified up to $20 \mathrm{dBm}$ before being launched at the $90 \%$ port of the coupler. The CW probe at $1559.1 \mathrm{~nm}$ is launched at the corresponding $10 \%$ port of the coupler. The combined signals are then launched into the HNLF. Polarization controllers (PCs) at each arm of the system are used to maximize the XPM effect in the HNLF. The fiber has a length of $310 \mathrm{~m}$, a dispersion of $-0.31 \mathrm{ps} / \mathrm{nm} / \mathrm{km}$, a dispersion slope of $0.0031 \mathrm{ps} / \mathrm{nm}^{2} / \mathrm{km}$, a nonlinear coefficient of $22 / \mathrm{W} / \mathrm{km}$ and a loss of $1.21 \mathrm{~dB} / \mathrm{km}$. Two Gaussian optical filters with FWHM of $0.5 \mathrm{~nm}$ and offset detuning of $\sim 1 \mathrm{~nm}$ relative to the $\mathrm{CW}$ carrier are used to select the wavelength converted signal. The system has also
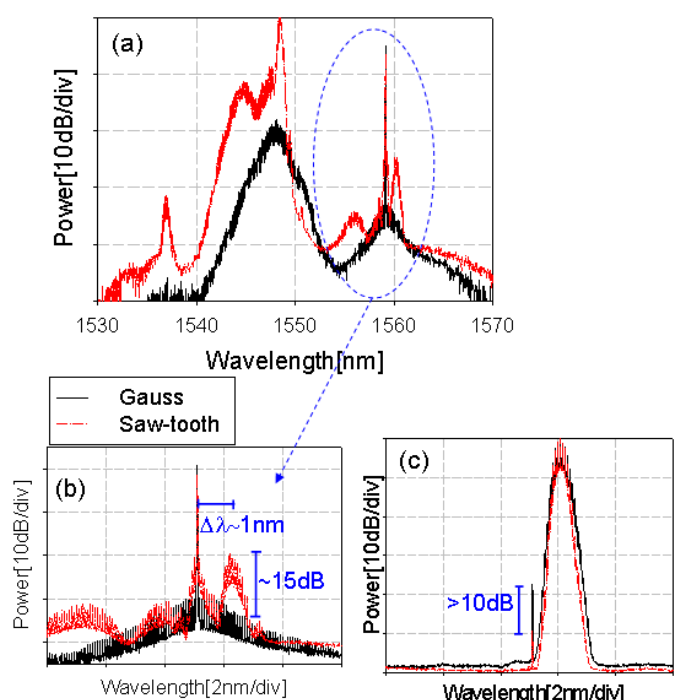

Figure 2. Spectral traces of the data signal at the output of the HNLF when Gaussian or saw-tooth pulses are used (a) and corresponding zoom-in around the $\mathrm{CW}$ wavelength (b). c) Spectral traces after the offset-filters when Gaussian or saw-tooth pulses are used.

been characterized without the SSFBG in place. In this case, to avoid the build-up of excessive SPM of the pump signal due to the much shorter duration of the unshaped pulses (FWHM of $1.3 \mathrm{ps}$ only), which can then overlap and interfere with the converted signal spectrum, its power was restricted to $5 \mathrm{dBm}$. The quality of the shaped pulses was monitored using an optical sampling oscilloscope (OSO), see Fig. 1(c) for the corresponding temporal profile. The pulse shape characteristics are in good agreements with the ones measured using a linear frequency-resolved optical gating, which are reported in [7]. As a reference, the measurement of the Gaussian input pulse is also shown in Fig. 1(b). Fig. 2(a) shows spectral traces after the HNLF when saw-tooth (red dashed trace) and Gaussian (black solid trace) pulses are used as the pump signals. As expected, for the case of an unshaped input, the XPMinduced sidebands on the $\mathrm{CW}$ broaden symmetrically about the original wavelength. On the other hand, when the sawtooth pump is used, the corresponding spectra broaden asymmetrically due to the asymmetric shape of the pump pulses, while maintaining a compact spectrum. This behavior is the key to the performance improvement offered by the saw-tooth shape, since it allows for easier separation of the converted signal from the remaining frequency components. Fig.2 (b) shows the zoomed spectra around the converted signal for both pumps: the spectral density of the wavelength shifted components is much higher when using saw-tooth pulses, achieving an OSNR improvement of about $15 \mathrm{~dB}$ relative to the unshaped Gaussian pulses. In this way, the wavelength converted signal can easily be filtered using conventional band-pass filters, while maintaining a good OSNR level at the converted signal wavelength. Fig.2 (c) shows the filtered spectra of the wavelength converted signal when Gaussian and saw-tooth pumps are used. As 


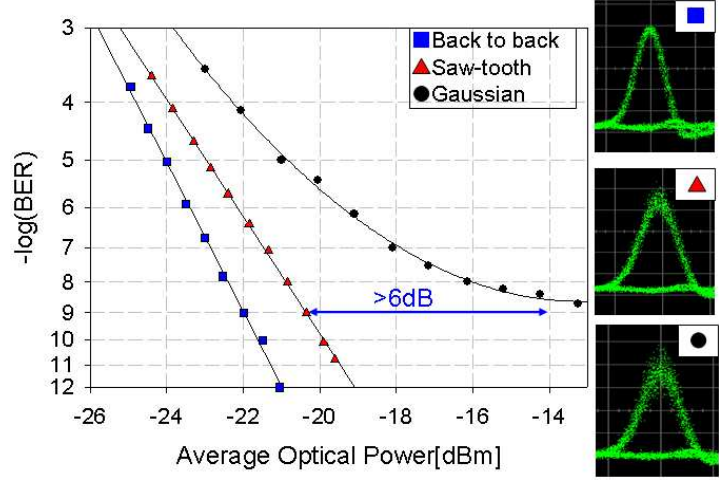

Figure 3. BER curves of the back-to-back original signal (blue rectangular symbols) and of the wavelength converted signal when saw-tooth (red triangular symbols) or Gaussian (black circle symbols) control pulses are employed. Inset: Corresponding eye-diagrams (Time scale:10ps/div).

can be seen from the figure, an improvement of more than $10 \mathrm{~dB}$ in the extinction ratio of the $\mathrm{CW}$ wave is achieved for saw-tooth pulses as compared to the Gaussian case.

To quantify the performance improvement offered by this system, we measured bit-error rate (BER) curves on the original and wavelength converted signals when either sawtooth or Gaussian pulses are used as the control signal. The corresponding curves are reported in Fig.3. A power penalty of $\sim 1.8 \mathrm{~dB}$ for error-free operation $\left(\mathrm{BER}=10^{9}\right.$ ) was achieved for saw-tooth pulses (red triangular symbols) as compared to the back-to-back original signal (blue rectangular symbols). When Gaussian pulses were used (black circles symbols), an error floor was clearly obtained in the BER curve and an additional power penalty of more than $6 \mathrm{~dB}$ incurred. For the interpretation of these power penalties, it is noted that no amplified spontaneous emission (ASE) rejection filter was used after the amplifier in the control path just before the HNLF. The impact of ASE can be appreciated by the severe error floor observed when Gaussian pump pulses are used. The corresponding eyediagrams for the back-to-back and wavelength converted signals are also shown in the inset of Fig. 3, when considering saw-tooth or Gaussian pulses. As a reflection of the BER measurements, when Gaussian pulses are used, some extra noise mainly on the "one level" can be seen on the eye, while good quality eye diagrams are obtained for saw-tooth pulses. Finally, it is worth pointing out that, in a wavelength conversion scheme using a $\mathrm{CW}$ beam as the input signal, the minimum achievable pulse width is limited by the temporal width of the pump (assuming the dispersion of the HNLF to be negligible as appropriate in our particular case) and the bandwidth of the optical offset filters. In our specific case, for Gaussian pulses, the relatively narrow filter bandwidth $(\sim 0.5 \mathrm{~nm})$ was the main cause of the temporal broadening of the wavelength converted pulses. On the other hand, for the saw-tooth pulses the temporal broadening was the result of the filter bandwidth as well as the broader pulse width of the new purpose-shaped pulses. This temporal broadening as compared to the data input signal can be qualitatively appreciated from the eye diagrams of Fig.3. This broadening could be easily avoided using shorter saw-tooth pulses and broader offset filters bandwidth.

\section{CONCLUSION}

In conclusion, we have demonstrated a wavelength conversion application which benefits from the constant time derivative profile of specifically designed saw-tooth pulses. The pulses were generated using pulse shaping in a SSFBG. The enhanced performance of the wavelength converter has been quantified through spectral and BER measurements. An OSNR improvement of the wavelength converted signal of about $15 \mathrm{~dB}$ when using saw-tooth control shape as compared to the use of more conventional pulse shapes, such as Gaussian pulses, has been shown, and an improvement in the receiver sensitivity of more than $6 \mathrm{~dB}$ has been achieved. We believe that this approach could be applied to much higher bit rate systems by properly scaling the width of the shaped pulses and the bandwidth and offset of the filter.

\section{ACKNOWLEDGEMENTS}

The authors acknowledge The Furukawa Electric Company, Japan for the loan of the HNLF and Alnair Labs, Japan for the loan of the optical sampling oscilloscope used in the experiment.

\section{REFERENCES}

[1] K.E. Stubkjaer, "Semiconductor optical amplifier-based all-optical gates for high-speed optical processing" IEEE J. Sel. Topics Quantum Electron., 6 (6), pp.1428-1435, 2000.

[2] K. Inoue and H. Toba, "wavelength conversion experiment using fiber four-wave mixing," IEEE Photon. Technol. Lett., vol.16,pp.6972, 1992.

[3] K. J. Blow, N.J. Doran, B. K. Nayar, B. P. Nelson, "Two-wavelength operation of the nonlinear fiber loop mirror", Opt. Lett., 15(4), pp. 248-250, 1990.

[4] B.-E. Olsson, P. Ohlen, L. Rau, D. J. Blumenthal, "A simple and robust $40-\mathrm{Gb} / \mathrm{s}$ wavelength converter using fiber cross-phase modulation and optical filetring“, IEEE Photon. Technol. Lett., 12, pp. 846-848, 2000.

[5] S. Watanabe, F. Futami, R. Okabe, Y. Takita, S. Ferber, R. Ludwig, C. Schubert, C. Schmidt, H. G. Weber, "160 Gb/s Optical 3RRegenerator in Fiber Transmission experiment", Proc. Of OFC, PD16-1, 2003.

[6] Vahid G. Ta'eed, Libin Fu, Mark Pelusi, Martin Rochette, Ian C. Littler, David J. Moss, and Benjamin J. Eggleton, "Error free all optical wavelength conversion in highly nonlinear As-Se chalcogenide glass fiber," Opt. Express 14, 10371-10376 (2006).

[7] F. Parmigiani, M. Ibsen, T. T. Ng, L. Provost, P. Petropoulos, D. J. Richardson, "An Efficient Wavelength Converter Exploiting a Grating-Based Saw-Tooth Pulse Shaper", IEEE Photon. Technol. Lett., 20 (17), pp.1461-1463, September 2008.

[8] F. Parmigiani, M. Ibsen, P. Petropoulos, D. J. Richardson, "Efficient All-Optical Wavelength Converter using saw-Tooth Pulses," CLEO/Europe-IQEC 2009 Munich.

[9] F. Parmigiani, T. T. Ng, M. Ibsen, P. Petropoulos, D. J. Richardson, "Timing jitter tolerant OTDM demultiplexing using a saw-tooth pulse shaper,” IEEE Photon. Technol. Lett. 20, 1992-1994 (2008).

[10] F. Parmigiani, P. Petropoulos, M. Ibsen, P. J. Almeida, T. T. Ng, and D. J. Richardson, "Time domain add-drop multiplexing scheme enhanced using a saw-tooth pulse shaper," Opt. Express 17, 83628369 (2009). 